\title{
Silicone oil removal after extended tamponade in proliferative diabetic retinopathy -a long range of follow-up
}

\author{
Mei-Chi Tsui $\mathbb{D}^{1} \cdot$ Yi-Ting Hsieh ${ }^{1} \cdot$ Chung-May Yang ${ }^{1,2}$
}

Received: 18 September 2019 / Revised: 30 January 2020 / Accepted: 6 February 2020 / Published online: 18 February 2020

(c) The Author(s), under exclusive licence to The Royal College of Ophthalmologists 2020

\begin{abstract}
Background To investigate the anatomical and functional results of silicone oil (SO) removal after an extended period of SO tamponade in eyes having received vitrectomy for proliferative diabetic retinopathy (PDR).

Methods From May 2009 to August 2017, clinical records of patients who had vitrectomy for PDR and underwent SO removal were retrospectively reviewed. SO was in principle left in the eye for an extended period of time and would be removed promptly when complications relevant to SO rose, or at the same setting when other intraocular surgeries were performed. Main outcome measures include anatomical outcome, functional outcome, and postoperative complications.

Results Seventy-four eyes of 64 patients (31 males and 33 females) were analysed. The mean follow-up duration was 35.6 months (ranging from 6 to 99 months, median 32 months). The duration of SO tamponade ranged from 3 to 116 months (mean 26.89 months, median 16 months). Anatomical success was achieved in $95.9 \%$ at the last follow-up with bestcorrected visual acuity (BCVA) becoming better or unchanged in $81.1 \%$. Postoperative complications included ocular hypertension ( $>25 \mathrm{mmHg}$; $>4$ weeks) in three eyes $(4.1 \%)$, macular hole in two eyes $(2.7 \%)$, transient choroidal detachment in one eye (1.4\%), vitreous haemorrhage in four eyes (5.4\%) and hyphaema in two eyes $(2.7 \%)$.

Conclusions The rate of retinal redetachment after an extended period of SO tamponade and removal was low and the majority of eyes obtained final visual acuity improvement. SO removal after an extended period of tamponade in PDR seems to be safe under proper indications and case selection.
\end{abstract}

\section{Introduction}

In the surgical treatment of complicated proliferative diabetic retinopathy (PDR), silicone oil (SO) may be used for long-term tamponade. In addition to early visual rehabilitation, the use of SO in PDR has the advantages of maintaining retinal attachment, lowering the rate and severity of recurrent bleeding and reducing the possibility of rubeosis iridis [1-10]. However, SO may also be associated with adverse effects including glaucoma, corneal oedema, cataract formation and peri-SO reproliferation [11-19]. Thus, it is a common practice to remove $\mathrm{SO}$ when an eye stays

Chung-May Yang

chungmay@ntu.edu.tw

1 Department of Ophthalmology, National Taiwan University Hospital, Taipei, Taiwan

2 Department of Ophthalmology, College of Medicine, National Taiwan University, Taipei, Taiwan attached for a few months after operation. However, early SO removal may run the risk of recurrent detachment, and inflammation induced by SO removal procedure may stimulate the development of rubeosis iridis [20, 21]. Although there are some studies on strategies of SO removal and on the rate of complications [6, 16, 17, 22-26], most of the reports focused on rhegmatogenous retinal detachment (RD) with proliferative vitreoretinopathy (PVR). The literature regarding SO removal in PDR is scanty and most studies were conducted decades ago $[2,6,10,19,27,28]$. The proper timing for SO removal after diabetic vitrectomy has not been well established. Strategies to reduce the complications after SO removal are also lacking. One reasonable approach is to withhold early SO removal, but to remove it promptly when complications relevant to SO rise, or at the same setting when other intraocular surgeries are performed. We adopted this strategy for SO removal after diabetic vitrectomy. Anatomical and functional outcomes and complications after SO removal are presented. 


\section{Materials and methods}

Medical records were retrospectively reviewed on consecutive patients with severe proliferative diabetic retinopathy who had previous pars plana vitrectomy (PPV) and had SO removed by the third author at National Taiwan University Hospital during the period of May 2009 to August 2017. We identified 74 eyes of 64 patients who had a minimum follow-up of 6 months after SO removal. All eyes were filled with 5000-centistoke (cs) SO in the previous surgery during the period of March 2003 to February 2017. Indications of SO injection were: (1) severe proliferation in four quadrants with fibrovascular tissue extending beyond the equator in more than two quadrants, and (2) the presence of residual traction or multiple breaks in different quadrants with suspected missed breaks after membrane dissection. Under these conditions, we believed that without long-term tamponade, the possibility of redetachment was high [29]. There were 105 eyes, which received diabetic vitrectomies with $\mathrm{SO}$ tamponade during the study period, and in 31 eyes (29.5\%), SO was left in situ. The reasons for retaining SO include: the retina was judged not suitable for SO removal for fear of redetachment or hypotony; prominent rubeosis with or without neovascular glaucoma was present; the visual potential was light perception or less; the duration of SO tamponade was $<9$ months; and finally, the patients opted not to have further surgery. This study was approved by the Research Ethics Committee of National Taiwan University Hospital and was conducted in accordance with the Declaration of Helsinki. Indications for SO removal were as follows: (1) massive preretinal blood or fibrin in the early post-operative period, (2) significant preretinal membrane causing macular structural changes and affecting central vision, (3) mature cataract or cataract causing progressive vision decrease, (4) intraocular pressure (IOP) higher than $25 \mathrm{mmHg}$ lasting for more than 4 weeks despite maximal antiglaucomatous eye drops, (5) progressive corneal endothelial cell loss, (6) significant emulsified $\mathrm{SO}$ in the anterior chamber (AC) causing decreased vision or increased IOP, (7) macular hole, and (8) on patient's request in a stable condition. As a principle, unless early intervention within a short period of time was required, at least 9 months was allowed before removing SO. Demographics (age, gender, and comorbidity), and ophthalmologic status before and after SO injection including best-corrected visual acuity (BCVA), IOP, lens status (phakic, pseudophakic, or aphakic), extent of fibrovascular proliferation (FVP) and RD were recorded. We defined FVP severity into four grades based on the severity of vitreoretinal adhesion: multiple-point adhesions with or without 1-site plaque-like broad adhesion (Grade 1), broad adhesions in more than 1 but fewer than three sites, located posterior to the equator (Grade 2), broad adhesions

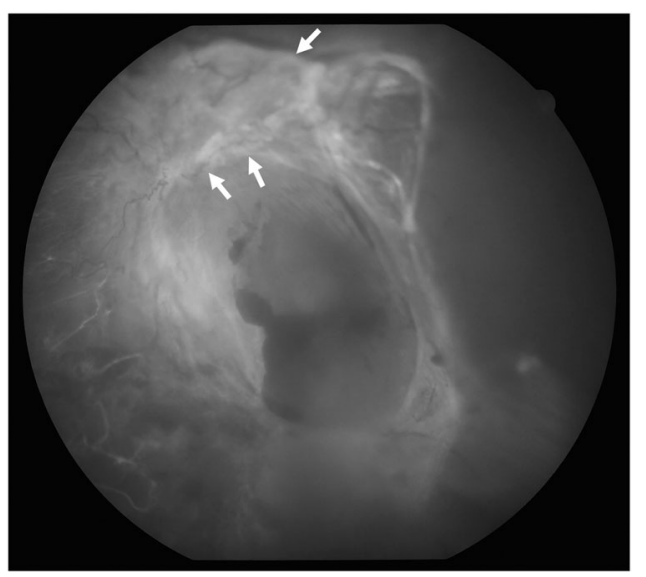

Fig. 1 Broad adhesion of fibrovascular proliferative membrane. The representative fundus photograph showing broad adhesion of fibrovascular proliferative membrane (arrows).

in more than three sites, located posterior to the equator or extending beyond the equator within 1 quadrant (Grade 3), and broad adhesions extending for more than 1 quadrant anterior to the equator (Grade 4) [30]. Broad adhesion is defined as fibrovascular proliferative membrane having multiple point adhesions and occupying more than two-disc areas (Fig. 1) [31]. The vitreoretinal status was assessed preoperatively with indirect ophthalmoscope or intraoperatively when media opacity obscured ophthalmoscopic examination.

\section{Surgical procedure}

Informed consent was obtained preoperatively. All surgeries were performed by the third author. SO removal of all patients was done by using a standard three-port PPV. AC irrigation to remove SO droplets was done if necessary. Visually significant cataract was removed, and intraocular lens was implanted when indicated. Retina was carefully examined after SO removal and supplement endolaser was performed if previous panretinal photocoagulation was judged insufficient. Epiretinal membrane (ERM) and internal limiting membrane (ILM) in the macular area were removed for premacular membrane causing structural changes. Repeated air-fluid exchange was done to remove residual SO followed by intravitreal injection (IVI) of bevacizumab $1.25 \mathrm{mg}$ in all eyes.

\section{Main outcome measures}

\section{Anatomical and functional outcomes}

Initial, early and final anatomical successes were defined and recorded respectively as complete retinal attachment at the first and third postoperative months and at the last follow-up. Functional outcomes were assessed by BCVA 
measured preoperatively, at the first and third months postoperatively, and at the last follow-up. Ambulatory vision was defined as $B C V A \geq 20 / 1000$.

\section{Complications}

Ocular hypertension was defined as IOP $>25 \mathrm{mmHg}$ and recorded as early postoperative (within 2 weeks after surgery), late-onset (after 2 weeks postoperatively), and prolonged ocular hypertension ( $>4$ weeks of IOP elevation despite full medication). Hypotony was defined as IOP $<$ $5 \mathrm{mmHg}$ for more than 1 week. Keratopathy, macular hole $(\mathrm{MH})$, cataract progression, posterior capsular opacity, vitreous haemorrhage $(\mathrm{VH})$, significant preretinal haemorrhage, and hyphaema were recorded.

\section{Statistical analysis}

Means and standard deviations were calculated for quantitative variables. Frequencies and percentages were calculated for categorical variables. Multivariate analysis of factors for the final visual outcome was performed. Logistic regression was used for investigating factors relevant to anatomical outcome. $P<0.05$ was considered statistically significant. The statistical analysis of the data was done by the software Statistical Package for Social Sciences (SPSS) version 22. Descriptive statistics was calculated in terms of mean, standard deviation, minimum, maximum and range.

\section{Results}

\section{Demographics}

Seventy-four eyes of 64 patients (31 males and 33 females) were analysed in this study. Patient ages ranged from 26 to 70 years (mean: $44.1 \pm 10.2$ years). Four patients were type 1 diabetes mellitus (DM), 55 patients were type $2 \mathrm{DM}$ and 5 were not documented. Three most common comorbidities were hypertension, diabetic kidney disease and heart disease in 42 patients ( 48 eyes), 14 patients ( 14 eyes), and 8 patients ( 8 eyes), respectively. Initially, 52 eyes had grade 4 FVP, 13 eyes had grade 3 FVP, 5 eyes had grade 2 FVP, 2 eyes had no FVP but macular hole, and 2 eyes had no documentation of FVP severity. Before SO injection, panretinal photocoagulation was performed for 53 eyes $(71.6 \%)$ and IVI bevacizumab $1.25 \mathrm{mg}$ was performed for 27 eyes (36.5\%). Tractional retinal detachment (TRD) in 31 eyes (41.9\%) and combined RD in 43 eyes (58.1\%) were initially diagnosed. Primary vitrectomy with SO injection was performed for 71 eyes and secondary vitrectomy with SO injection was performed for the other 3 eyes with failed initial vitrectomy with gas tamponade.

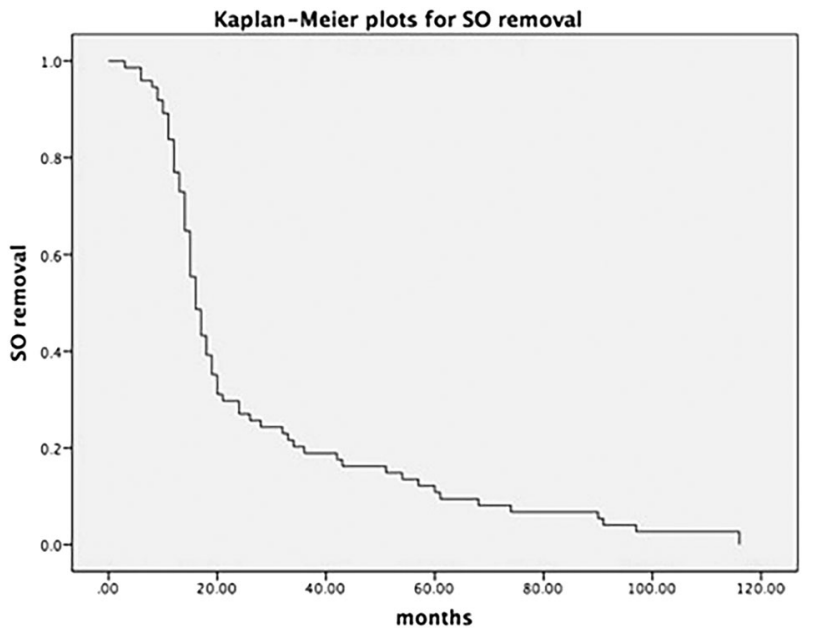

Fig. 2 Kaplan-Meier plot. Kaplan-Meier plot for silicone oil removal.

Table 1 Indications for silicone oil removal.

\begin{tabular}{|c|c|c|}
\hline & $\begin{array}{l}\text { No. of } \\
\text { eyes }^{\text {a }}(n)\end{array}$ & $\begin{array}{l}\text { Average duration } \\
\text { (months) }\end{array}$ \\
\hline $\begin{array}{l}\text { Massive preretinal blood or fibrin in } \\
\text { the early post-operative period }\end{array}$ & 1 & 3.0 \\
\hline $\begin{array}{l}\text { Macular pucker causing structural } \\
\text { changes }\end{array}$ & 25 & 31.3 \\
\hline Along with cataract operation & 51 & 16.7 \\
\hline Ocular hypertension & 5 & 36.2 \\
\hline $\begin{array}{l}\text { Significant amount of emulsified } \\
\text { oil in AC }\end{array}$ & 23 & 40.9 \\
\hline Macular hole & 2 & 13.5 \\
\hline $\begin{array}{l}\text { On patient's request with stable } \\
\text { retinal conditions }{ }^{\mathrm{b}}\end{array}$ & 2 & 15.5 \\
\hline
\end{tabular}

$A C$ anterior chamber, $S O$ silicone oil.

${ }^{\text {a }}$ The total number of eyes exceeds 74 because some patients had multiple reasons for SO removal.

${ }^{\mathrm{b}}$ Stable retinal conditions are defined as complete flat retina for more than 9 months without vitreoretinal traction or residual preretinal membrane with surrounding laser scars and no retinal folding.

\section{Indications for SO removal}

Twenty-one eyes (28.4\%) were pseudophakic, and all the others were phakic. Before SO removal, retina was attached in all eyes. The duration of SO tamponade ranged from 3 to 116 months (mean: 26.89 \pm 25.45 months, median: 16 months). Kaplan-Meier plot for SO removal is demonstrated in Fig. 2. Indications for SO removal are listed in Table 1. Only one eye (1.4\%) had a SO tamponade duration $<6$ months and in this case, SO removal was indicated due to massive preretinal fibrin from recurrent preretinal haemorrhage after initial surgery, along with hyphaema and persistent ocular hypertension. The follow-up duration after SO removal ranged from 6 to 99 months (mean: $35.55 \pm$ 
21.82 months, median: 32 months). As for indications, those with significant emulsified oil in AC had a longer duration of SO tamponade before removal, while those receiving SO removal due to cataract progression had a shorter duration of SO tamponade before removal $(p=$ 0.019 by ANOVA).

\section{Main outcome measures}

\section{Anatomical outcomes}

Anatomical success was achieved in 72 eyes (97.3\%) at one month and three months after SO removal and in 71 eyes $(95.9 \%)$ at the last follow-up visit. Retinal redetachment after SO removal occurred in 3 eyes (4.1\%); two of them had recurrent FVP and TRD at, respectively, 1 month and 7 months after SO removal, and SO was reinjected in both cases. The other one had macular hole with retinal detachment (MHRD) immediately after SO removal, and it then received PPV along with ILM removal and inverted ILM flap insertion procedures 1 month later. All of these three eyes with retinal redetachment after SO removal had attached retina after the secondary operations. Logistic regression models were used to evaluate the risk factors for redetachment of retina after SO removal, and a lower IOP before SO removal was identified as the only variable relevant to final anatomical success $(\mathrm{OR}=0.775, p=$ 0.038) (Table 2). Overall, SO free with reattached retina was achieved in 72 eyes $(97.3 \%)$.

\section{Visual outcomes}

After SO removal, BCVA improved significantly from LogMAR $1.53 \pm 0.54$ to $1.18 \pm 0.58$ at 1 month $(p<0.001)$, $1.14 \pm 0.62(p<0.001)$ at 3 months after SO removal and $1.01 \pm 0.61(p<0.001)$ at the last follow-up visit. The correlation between preoperative and final BCVAs is demonstrated in Fig. 3. In eyes without combined cataract operation, BCVA also improved significantly from logMAR 1.46 to $1.15(p<0.001)$ at the last follow-up visit. Forty-nine eyes $(66.2 \%)$ achieved a final visual acuity of $20 / 200$ or better. BCVA improved or was stable in 56 eyes (75.7\%) 3 months postoperatively and in 60 eyes $(81.1 \%)$ at the last follow-up. Seven eyes $(9.5 \%)$ had worsened vision but maintained an ambulatory vision, and 1 eye (1.4\%) had no light perception at the last follow-up.

Under univariate analysis, initial FVP grade 3 ( $p=$ $0.009)$, initial RD in three quadrants $(p=0.049)$ and in all four quadrants $(p<0.001)$, BCVA before SO injection $(p=$ $0.021)$, and BCVA before SO removal $(p<0.001)$ were identified as variables associated with final BCVA (Table 3). For multivariate analysis of the five variables, only BCVA before SO injection (beta $=0.294, p=0.005$ )
Table 2 Correlating factors for retinal attachment after silicone oil removal by using logistic regression analysis.

\begin{tabular}{|c|c|c|c|c|}
\hline & \multirow[t]{2}{*}{$P$ value } & \multirow[t]{2}{*}{ OR } & \multicolumn{2}{|l|}{ CI $95 \%$} \\
\hline & & & Lower & Upper \\
\hline Age & 0.388 & 0.946 & 0.835 & 1.073 \\
\hline Gender & 0.563 & 0.057 & 0.042 & 5.606 \\
\hline Combined RD & 0.803 & 1.366 & 1.118 & 15.798 \\
\hline FVP grade & 0.840 & 1.180 & 0.238 & 5.842 \\
\hline RD quadrants & 0.365 & 0.529 & 0.134 & 2.095 \\
\hline Pre-op PRP & 0.149 & 0.163 & 0.014 & 1.917 \\
\hline Pre-op IVIA & 0.908 & 0.865 & 0.075 & 10.014 \\
\hline BCVA before SO injection & 0.220 & 0.216 & 0.019 & 2.502 \\
\hline IOP before SO injection & 0.828 & 0.963 & 0.685 & 1.354 \\
\hline CMT before SO injection & 0.409 & 0.980 & 0.933 & 1.029 \\
\hline BCVA before SO removal & 0.992 & 1.011 & 0.117 & 8.772 \\
\hline IOP before SO removal & 0.038 & 0.775 & 0.608 & 0.986 \\
\hline $\begin{array}{l}\text { Macular oedema before SO } \\
\text { removal }\end{array}$ & 0.320 & 0.289 & 0.025 & 3.343 \\
\hline CMT before SO removal & 0.395 & 0.979 & 0.932 & 1.028 \\
\hline Lens status (pseudophakia) & 0.889 & 1.190 & 0.102 & 13.850 \\
\hline SO amount & 0.238 & 2.420 & 0.558 & 10.489 \\
\hline Duration of SO tamponade & 0.752 & 0.991 & 0.935 & 1.049 \\
\hline Combine with cataract operation & 0.931 & 0.898 & 0.077 & 10.433 \\
\hline $\begin{array}{l}\text { Combine with ERM/ILM } \\
\text { peeling }\end{array}$ & 0.973 & 0.958 & 0.083 & 11.121 \\
\hline Emulsified oil in AC & 0.213 & 4.762 & 0.409 & 55.402 \\
\hline $\begin{array}{l}\text { BCVA } 1 \text { month after SO } \\
\text { removal }\end{array}$ & 0.595 & 0.547 & 0.059 & 5.051 \\
\hline IOP 1 month after SO removal & 0.433 & 0.897 & 0.683 & 1.177 \\
\hline Final BCVA & 0.969 & 0.963 & 0.140 & 6.638 \\
\hline BCVA change & 0.962 & 0.954 & 0.139 & 6.553 \\
\hline CMT at last follow-up & 0.297 & 1.016 & 0.986 & 1.046 \\
\hline Hyphaema & 0.999 & 0.000 & 0.000 & - \\
\hline $\mathrm{VH}$ & 0.074 & 11.333 & 0.789 & 162.743 \\
\hline Follow-up duration & 0.384 & 0.968 & 0.899 & 1.042 \\
\hline
\end{tabular}

$A C$ anterior chamber, $B C V A$ best-corrected visual acuity, $C I$ confidence interval, $C M T$ central macular thickness, ERM epiretinal membrane, $F V P$ fibrovascular proliferation, ILM internal limiting membrane, IOP intraocular pressure, IVIA intravitreal injection of Avastin, $O R$ odds ratio, Pre-op preoperative, $P R P$ panretinal photocoagulation, $R D$ retinal detachment, $S O$ silicone oil, $V H$ vitreous haemorrhage.

$P<0.05$ was considered statistically significant and values which achieved statistical significance were marked in bold types.

and BCVA before SO removal (beta $=0.466, p<0.001$ ) were identified as variables associated with visual outcome.

\section{Complications}

Two eyes $(2.7 \%)$ had immediate postoperative ocular hypertension $(>25 \mathrm{mmHg}$ ) while eight eyes $(10.8 \%)$ had late-onset IOP elevation after SO removal. Three eyes 
correlation of pre-op and post-op BCVA

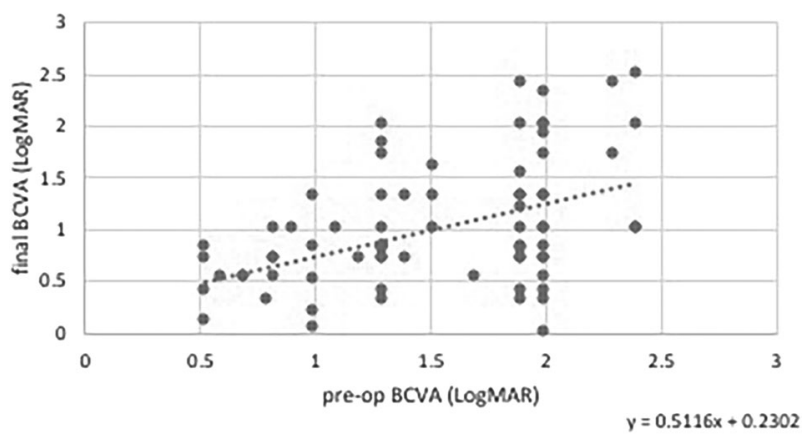

Fig. 3 Preoperative and final best-corrected visual acuity. The correlation of preoperative and final best-corrected visual acuity of the total 74 eyes in this study.

(4.1\%) developed de novo long-term ocular hypertension (lasting for more than 4 weeks despite full medication), among which one received peripheral iridectomy and the other two were controlled with medications. No eye had hypotony $(<5 \mathrm{mmHg})$ postoperatively, but transient low IOP $(<10 \mathrm{mmHg})$ was found in six eyes $(8.1 \%)$. Both of the only two phakic eyes showed cataract progression and received cataract operation 11 months and 57 months after SO removal, respectively. Posterior capsular opacity developed in $24(33.3 \%)$ of the 72 pseudophakic eyes. Immediately after SO removal, one eye (1.4\%) had transient choroidal detachment, which resolved on postoperative day 4 under oral and topical steroid. Two eyes (2.7\%) had full thickness macular hole (FTMH) after SO removal; one of them had FTMH with RD immediately after SO removal and received PPV along with ILM removal, inverted ILM flap insertion procedures 1 month postoperatively as described previously; the other had FTMH without RD 2 weeks after SO removal and received PPV and posterior lens capsule insertion 16 months after SO removal. Macular hole closure was achieved postoperatively and the retina remained attached in both cases. Four eyes (5.4\%) had recurrent $\mathrm{VH}$ immediately after $\mathrm{SO}$ removal and received IVI bevacizumab with or without cryotherapy at 1, 1, 4, 5 months, respectively. Among them, one had complicated recurrent FVP and TRD and received PPV again with SO reinjection. The fundus was clear in these four eyes postoperatively and remained clear until the last follow-up visit. Two eyes $(2.7 \%)$ had recurrent hyphaema and received AC irrigation at 1 week and 2 months, respectively. None of the 74 eyes developed keratopathy.

\section{Discussion}

In this study, results of SO removal after an extended period of SO tamponade were analysed. We withheld SO removal for at least 9 months and until other intraocular procedures
Table 3 Correlating factors for final best-corrected visual acuity after silicone oil removal by using linear regression analysis.

\begin{tabular}{|c|c|c|}
\hline & Beta & $P$ value \\
\hline Age & 0.202 & 0.084 \\
\hline Gender & -0.099 & 0.401 \\
\hline Combine RD & 0.126 & 0.291 \\
\hline \multicolumn{3}{|l|}{ FVP grade } \\
\hline \multicolumn{3}{|l|}{ Grade 2} \\
\hline Grade 3 & -0.595 & 0.009 \\
\hline Grade 4 & -0.138 & 0.307 \\
\hline \multicolumn{3}{|l|}{ RD extent } \\
\hline \multicolumn{3}{|l|}{2 quadrants } \\
\hline 3 quadrants & 0.306 & 0.049 \\
\hline 4 quadrants & 0.089 & $<\mathbf{0 . 0 0 1}$ \\
\hline Pre-op PRP & -0.189 & 0.112 \\
\hline Pre-op IVIA & -0.147 & 0.212 \\
\hline BCVA before SO injection & 0.272 & 0.021 \\
\hline IOP before SO injection & 0.067 & 0.579 \\
\hline CMT before SO injection & -0.092 & 0.589 \\
\hline BCVA before SO removal & 0.454 & $<0.001$ \\
\hline IOP before SO removal & 0.084 & 0.481 \\
\hline Macular oedema before SO removal & 0.117 & 0.387 \\
\hline CMT before SO removal & 0.077 & 0.598 \\
\hline Lens status (pseudophakia) & 0.023 & 0.846 \\
\hline SO amount & 0.200 & 0.093 \\
\hline Duration of SO tamponade & -0.004 & 0.970 \\
\hline Combine with cataract operation & -0.113 & 0.338 \\
\hline Combine with ERM/ILM peeling & 0.181 & 0.330 \\
\hline Emulsified oil in AC & 0.079 & 0.608 \\
\hline $\mathrm{CMT}$ at last $\mathrm{f} / \mathrm{u}$ & -0.183 & 0.180 \\
\hline Recurrent RD & -0.005 & 0.970 \\
\hline Hyphaema & 0.110 & 0.350 \\
\hline VH & 0.076 & 0.517 \\
\hline Laser capsulotomy & -0.218 & 0.062 \\
\hline Follow-up duration & -0.021 & 0.857 \\
\hline
\end{tabular}

$A C$ anterior chamber, $B C V A$ best-corrected visual acuity, $C M T$ central macular thickness, $F V P$ fibrovascular proliferation, $f / u$ follow-up, ERM epiretinal membrane, ILM internal limiting membrane, IOP intraocular pressure, IVIA intravitreal injection of Avastin, Pre-op preoperative, $P R P$ panretinal photocoagulation, $R D$ retinal detachment, $S O$ silicone oil, $V H$ vitreous haemorrhage.

$P<0.05$ was considered statistically significant and values which achieved statistical significance were marked in bold types.

were indicated. The anatomical results were favourable (more than $95 \%$ of eyes had attached retina after SO removal); functional results and complications were also acceptable as compared with other available study results. The reason we adopted the time period of at least 9 months after surgery to remove SO in uncomplicated cases instead of 3-6 months as advocated by other investigators $[17,26,32]$ is that after intraocular surgeries in eyes with 
PDR, postoperative inflammation was strong [33] and it usually took a prolonged period of time to obtain a complete subsidence of inflammation [34, 35]. Our results indicate that this strategy of timing for SO removal from eyes with PDR may be desirable in the modern surgical era.

Retinal redetachment is one of the most serious complications after SO removal. Literature review showed in different underlying conditions, the rate of retinal redetachment varied widely and appeared to correlate with the underlying disease process and its severity. In RD with PVR eyes, retinal redetachment rate occurred in 20\% [36] and up to $51.5 \%$ of cases [27]. Redetachment following the removal of SO was less common in diabetic eyes, ranging from 11 to $31 \%$, with an average duration of SO tamponade being 19 weeks to 13.5 months [2, 10, 19, 27, 28]. In our study, three eyes $(4.1 \%)$ had retinal detachment after SO removal with an average tamponade duration of 26.9 months, and this rate is much less than those reported in previous studies. Improvements in surgical techniques and instruments, as well as the use of anti-vascular endothelial growth factor agents may play important roles in lowering the redetachment rate since most of the other studies were conducted decades ago. Further, the mean

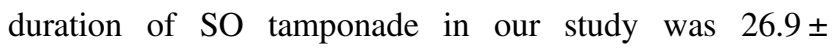
25.4 months (ranging from 3 to 116 months, median 16 months), which was by far the longest duration of SO tamponade among the relevant studies. We believe that an extended period of SO tamponade may have the following advantages after SO removal surgery: stabilising the retina, reducing the possibility of excessive post-operative inflammation, and rendering the residual fibrotic tissue less contractile. These advantages may decrease the likelihood of redetachment. In our study, it is found that IOP before SO removal is the only variable relevant to the final anatomical success. Eyes with low IOP before SO removal had a higher risk of recurrent retinal detachment. Two of the three retinal redetachments occurred in the first month after SO removal. These three cases with redetachment were manageable and the retinas remained attached after operation. The functional prognosis of recurrent detachment was satisfactory with a mean BCVA of $\log$ MAR 1.0, and is superior to that previously reported [36-38].

Under our indications for SO removal with a relatively extended period of tamponade, we found that there is no correlation between the duration of SO tamponade and the incidence of redetachment. Those three cases with RD after SO removal had SO tamponade for 14, 20, and 33 months, respectively. Two eyes had incomplete FVP removal during the two previous operations, and the other had MHRD.

In our study, patients' vision improved significantly after SO removal. Sixty eyes $(81.1 \%)$ had improved or stable BCVA at the last follow-up, and 49 eyes (66.2\%) achieved a final visual acuity of $20 / 200$ or better. These results compare favourably with those of other previous studies. Again, these results indicate the benefit of improved surgical techniques and instruments. To the best of our knowledge, the data we present are the first surgical results in the modern era of micro-invasive surgery. In addition, we did not find any association between the final BCVA and the duration of SO tamponade in this study, and this suggests that an extended period of SO tamponade in the vitreous cavity may not cause retinal toxicity. However, we identified BCVA before SO injection and BCVA before SO removal as the variables associated with visual outcome through multivariate analysis. This result implies that the primary vitrectomy should be performed promptly once a patient has FVP and TRD threatening central vision.

In this study, one eye had early SO removal because of massive preretinal haemorrhage/fibrin. The fibrin clot was extensive and adherent to the vessels. Our previous experience suggests massive preretinal haemorrhagic mound should be removed promptly because if removal of SO and fibrin was delayed, TRD may develop in a short period of time.

One of the concerns of an extended period of SO tamponade in the vitreous cavity is the possibility of ocular hypertension secondary to SO droplets obstructing the trabecular meshwork. We removed $\mathrm{SO}$ once the antiglaucomatous medications failed to bring the IOP down to the normal range for more than one month. Overall, IOP decreased significantly from $18.3 \pm 6.4 \mathrm{mmHg}$ to $14.5 \pm 4.5 \mathrm{mmHg}$ after SO removal $(p<0.001)$ in this study. Of the five eyes that had IOP elevation before SO removal, all experienced a decrease in IOP after SO removal. However, one of the five eyes had an increase of IOP 15 months after SO removal and underwent successful trabeculectomy. The patient had been diagnosed as primary open-angle glaucoma, and the use of SO might also play some role in the impaired aqueous outflow system. Two eyes $(2.7 \%)$ had immediate postoperative IOP elevation $(>25 \mathrm{mmHg})$, and one of them was associated with hyphaema. Eight eyes $(10.8 \%)$ had late-onset transient IOP elevation, and among them, one was accompanied with hyphaema, and two were due to pupillary block. Three eyes $(4.1 \%)$ developed de novo long-term ocular hypertension (lasting for more than 4 weeks despite medication), and this rate is comparable with previous study [17]. For these three eyes, SO was removed at 15, 54, and 61 months, respectively, after SO injection. It is suspected that the high IOP was caused by the damage of trabecular meshwork by the SO droplets, although no grossly visible $\mathrm{SO}$ emulsification in the $\mathrm{AC}$ was noted.

In this study, significant emulsification of $\mathrm{SO}$ in the $\mathrm{AC}$ was found in 23 eyes $(31.1 \%)$ before SO removal in this study. Emulsification of the SO has been associated with a rise in IOP $[39,40]$. However, only 2 of the 23 eyes $(8.7 \%)$ 
with emulsification of SO developed persistent IOP elevation before SO removal in this study, and this suggests our timing of SO removal seems to be adequate.

Intraocular bleeding including vitreous haemorrhage and hyphaema is an infrequent but significant complication after SO removal, since it would affect IOP and markedly delay the visual recovery. In our study, $5.4 \%$ of eyes had VH and $2.7 \%$ had hyphaema. Patients with VH and hyphaema after SO removal in our series had a significantly shorter duration of SO tamponade than those without. This suggests that an extended duration of SO tamponade may prevent postoperative hyphaema and VH. Furthermore, some believed that SO had a positive effect against iris neovascularization [7-9]. Although no neovascularization was found in our study, it may be an important factor of postoperative $\mathrm{VH}$ and hyphaema. An extended duration of SO tamponade should be considered for patients with high ischaemic drive which makes neovascularization more likely to occur.

FTMH was found in two eyes (2.7\%) after SO removal in this study and in both cases, no detectable FVP was noted at the time of SO removal or after SO removal. Several studies reported MHs in diabetic retinopathy (DR) with or without FVP [41, 42]. To the best of our knowledge, we are the first to report development of $\mathrm{MH}$ after SO removal in DR. During reoperation, both of the two eyes had foveal thinning, and ERM in the upper and lower arcades and connected with vessels was found in one of them. We believe that the tangential traction from foveal thinning and ERM were responsible for the MH formation.

We acknowledge the limitations associated with a retrospective review of cases, including a lack of study control and standardised reporting of cases. In addition, it is difficult to distinguish whether most complications arising from $\mathrm{SO}$ removal, the previous use of SO, or the underlying retinal diseases. However, because there is no such study in modern micro-invasive surgery era, our study may be used as a base for future comparison. Our study shows a stable visual outcome throughout an extended follow-up, and minor and manageable complications associated with longterm SO usage and after SO removal. With understanding of possible complications and careful case selection, it is concluded that SO removal after a relatively extended period of SO tamponade and under proper indications after diabetic vitrectomy may be an acceptable option.

\section{Summary}

\section{What was known before}

- Silicone oil (SO) may be used for long-term tamponade in complicated proliferative diabetic retinopathy (PDR) but it may also be associated with adverse effects.
- The proper timing for SO removal after diabetic vitrectomy has not been well established and strategies to reduce the complications after SO removal are lacking.

- The literature regarding SO removal in PDR is scanty and most studies were conducted decades ago.

\section{What this study adds}

- The rate of retinal redetachment after an extended period of SO tamponade and removal was low and the majority of eyes obtained final visual acuity improvement.

- To withhold SO removal until other intraocular surgeries are performed or complications related to SO rise may be an acceptable option in the modern surgical era.

- As no study of SO removal in PDR in modern microinvasive surgery era has been reported, our study may be used as a base for future comparison.

\section{Compliance with ethical standards}

Conflict of interest The authors declare that they have no conflict of interest.

Publisher's note Springer Nature remains neutral with regard to jurisdictional claims in published maps and institutional affiliations.

\section{References}

1. Cibis PA, Becker B, Okun E, Canaan S. The use of liquid silicone in retinal detachment surgery. Arch Ophthalmol. 1962;68:590-9.

2. Pearson RV, McLeod D, Gregor ZJ. Removal of silicone oil following diabetic vitrectomy. Br J Ophthalmol. 1993;77:204-7.

3. McLeod D. Silicone-oil injection during closed microsurgery for diabetic retinal detachment. Graefes Arch Clin Exp Ophthalmol. 1986;224:55-59.

4. Yeo JH, Glaser BM, Michels RG. Silicone oil in the treatment of complicated retinal detachments. Ophthalmology. 1987; 94:1109-13.

5. The Silicone Study Group. Vitrectomy with silicone oil or sulfur hexafluoride gas in eyes with severe proliferative vitreoretinopathy: results of a randomized clinical trial. Silicone Study Report 1. Arch Ophthalmol. 1992;110:770-9.

6. Azen SP, Scott IU, Flynn HW Jr., Lai MY, Topping TM, Benati $\mathrm{L}$, et al. Silicone oil in the repair of complex retinal detachments. A prospective observational multicenter study. Ophthalmology. 1998;105:1587-97.

7. Ando F. Usefulness and limit of silicone in management of complicated retinal detachment. Jpn J Ophthalmol. 1987;31: 138-46.

8. Gabel VP, Kampik A, Gabel C, Spiegel D. Silicone oil with high specific gravity for intraocular use. Br J Ophthalmol. 1987; 71:262-7.

9. Lucke KH, Foerster MH, Laqua H. Long-term results of vitrectomy and silicone oil in 500 cases of complicated retinal detachments. Am J Ophthalmol. 1987;104:624-33. 
10. Silicone Oil Study Group of Sankara Nethralaya. Use of silicone oil in the management of complex retinal detachment-an Indian experience. Int Ophthalmol. 2004;25:129-42.

11. McCuen BW 2nd, Landers MB 3rd, Machemer R. The use of silicone oil following failed vitrectomy for retinal detachment with advanced proliferative vitreoretinopathy. Ophthalmology. 1985;92:1029-34.

12. Grey RH, Leaver PK. Silicone oil in the treatment of massive preretinal retraction. I. Results in 105 eyes. $\mathrm{Br} \mathrm{J}$ Ophthalmol. 1979;63:355-60.

13. Leaver PK, Grey RH, Garner A. Silicone oil injection in the treatment of massive preretinal retraction. II. Late complications in 93 eyes. Br J Ophthalmol. 1979;63:361-7.

14. Haut J, Ullern M, Chermet M, Van Effenterre G. Complications of intraocular injections of silicone combined with vitrectomy. Ophthalmologica. 1980;180:29-35.

15. Cox MS, Trese MT, Murphy PL. Silicone oil for advanced proliferative vitreoretinopathy. Ophthalmology. 1986;93:646-50.

16. Casswell AG, Gregor ZJ. Silicone oil removal. I. The effect on the complications of silicone oil. Br J Ophthalmol. 1987;71:893-7.

17. Casswell AG, Gregor ZJ. Silicone oil removal. II. Operative and postoperative complications. Br J Ophthalmol. 1987;71:898-902.

18. Shen YD, Yang CM. Extended silicone oil tamponade in primary vitrectomy for complex retinal detachment in proliferative diabetic retinopathy: a long-term follow-up study. Eur J Ophthalmol. 2007;17:954-60.

19. Gonvers M. Temporary silicone oil tamponade in the management of retinal detachment with proliferative vitreoretinopathy. Am J Ophthalmol. 1985;100:239-45.

20. McCuen BW 2nd, Rinkoff JS. Silicone oil for progressive anterior ocular neovascularization after failed diabetic vitrectomy. Arch Ophthalmol. 1989;107:677-82.

21. Heimann K, Dahl B, Dimopoulos S, Lemmen KD. Pars plana vitrectomy and silicone oil injection in proliferative diabetic retinopathy. Graefes Arch Clin Exp Ophthalmol. 1989;227:152-6.

22. Kampik A, Gandorfer A. Silicone oil removal strategies. Semin Ophthalmol. 2000;15:88-91.

23. Bassat IB, Desatnik H, Alhalel A, Treister G, Moisseiev J. Reduced rate of retinal detachment following silicone oil removal. Retina. 2000;20:597-603.

24. Falkner CI, Binder S, Kruger A. Outcome after silicone oil removal. Br J Ophthalmol. 2001;85:1324-7.

25. Federman JL, Schubert HD. Complications associated with the use of silicone oil in 150 eyes after retina-vitreous surgery. Ophthalmology. 1988;95:870-6.

26. Jonas JB, Budde WM, Knorr HL. Timing of retinal redetachment after removal of intraocular silicone oil tamponade. Am J Ophthalmol. 1999;128:628-31.
27. Kampik A, Hoing C, Heidenkummer HP. Problems and timing in the removal of silicone oil. Retina. 1992;12(3 Suppl):S11-16.

28. Jonas JB, Knorr HL, Rank RM, Budde WM. Retinal redetachment after removal of intraocular silicone oil tamponade. Br J Ophthalmol. 2001;85:1203-7.

29. Yeh PT, Yang CM, Yang CH. Distribution, reabsorption, and complications of preretinal blood under silicone oil after vitrectomy for severe proliferative diabetic retinopathy. Eye. 2012;26:601-8.

30. Chen SN, Yang CM. Internal limiting membrane flaps for coexistent macular hole and retinal detachment in eyes with proliferative diabetic retinopathy. J Ophthalmol. 2018;2018:3470731.

31. Sun JK, Silva PS, Cavallerano JD, Blodi BA, Davis MD, Aiello LM et al. (eds). Ryan's retina, 6th edn. Elsevier; 2017, p. 3305-98.

32. Zilis JD, McCuen BW 2nd, de Juan E Jr., Stefansson E, Machemer R. Results of silicone oil removal in advanced proliferative vitreoretinopathy. Am J Ophthalmol. 1989;108:15-21.

33. Ferguson VM, Spalton DJ. Continued breakdown of the blood aqueous barrier following cataract surgery. $\mathrm{Br} \mathrm{J}$ Ophthalmol. 1992;76:453-6.

34. Jeng CJ, Hsieh YT, Yang CM, Yang CH, Lin CL, Wang IJ. Development of diabetic retinopathy after cataract surgery. PLoS ONE. 2018;13:e0202347.

35. Liu Y, Luo L, He M, Liu X. Disorders of the blood-aqueous barrier after phacoemulsification in diabetic patients. Eye. 2004;18:900-4.

36. Hutton WL, Azen SP, Blumenkranz MS, Lai MY, McCuen BW, Han DP, et al. The effects of silicone oil removal. Silicone Study Report 6. Arch Ophthalmol. 1994;112:778-85.

37. Scholda C, Egger S, Lakits A, Haddad R. Silicone oil removal: results, risks and complications. Acta Ophthalmol Scand. 1997;75:695-9.

38. Franks WA, Leaver PK. Removal of silicone oil-rewards and penalties. Eye. 1991;5(Pt 3):333-7.

39. Riedel KG, Gabel VP, Neubauer L, Kampik A, Lund OE. Intravitreal silicone oil injection: complications and treatment of 415 consecutive patients. Graefes Arch Clin Exp Ophthalmol. 1990;228:19-23.

40. Ichhpujani P, Jindal A, Jay Katz L. Silicone oil induced glaucoma: a review. Graefes Arch Clin Exp Ophthalmol. 2009;247:1585-93.

41. Yeh PT, Cheng CK, Chen MS, Yang CH, Yang CM. Macular hole in proliferative diabetic retinopathy with fibrovascular proliferation. Retina. 2009;29:355-61.

42. Lai CT, Yang CM. Development of macular holes in diabetic retinopathy with fibrovascular proliferation: report of four cases. Taiwan J Ophthalmol. 2015;5:85-89. 